\title{
Inductance Formula for Square Planar Spiral Inductors with Rectangu- lar Conductor Cross Section
}

\author{
H. A. Aebischer ${ }^{1 *}$ \\ ${ }^{1}$ LEGIC Identsystems AG, Switzerland \\ *corresponding author, E-mail: hubert.aebischer@legic.com
}

\begin{abstract}
Planar spiral coils are used as inductors in radio frequency (RF) microelectronic integrated circuits (IC's) and as antennas in both $13.56 \mathrm{MHz}$ radio frequency identification (RFID) and telemetry systems. They must be designed to a specified inductance. From the literature, approximate formulae for the inductance of such coils with rectangular conductor cross section are known. They yield the direct current (DC) inductance, which is considered as a good approximation for inductors in RF IC's up to the GHz range. In principle, these formulae can simplify coil design considerably. But a recent comparative study of the most cited formulae [1] revealed that their maximum relative error is often much larger than claimed by the author, and too large to be useful in circuit design.

This paper presents a more accurate formula for the DC inductance of square planar spiral coils than was known so far. It is applicable to any design of such coils with up to 20 windings. Owing to its scalability, this holds irrespectively of the coil size and the inductance range. It lowers the maximum error over the whole domain of definition from so far $29 \%$ down to $2.0 \%$. This has been tested by the same method used in the comparative study [1], where the precise reference inductances were computed with the help of the free standard software FastHenry2. A comparison to measurements is included. Moreover, the source code of a MATLAB ${ }^{\circledR}$ function to implement the formula is given in the appendix.
\end{abstract}

[1] H. A. Aebischer, Advanced Electromagnetics, vol. 7, no. 5, 37-48, 2018.

\section{Introduction}

Planar spiral inductors are used in radio frequency (RF) microelectronic integrated circuits (IC's) [2] and both as $13.56 \mathrm{MHz}$ radio frequency identification (RFID) [3] and telemetry antennas [4]. In these applications, the coil must be designed to a predefined inductance. Therefore, values of the design parameters resulting in the specified inductance must be found. This represents a simple form of an inverse problem. It can only be solved indirectly, by using a method to calculate the inductance and by varying the parameters repeatedly, subject to any constraints, until the result matches the prescribed inductance to sufficient precision. Thereby, some method is needed to calculate the inductance of a coil from its geometric design parameters, as e.g. implemented in numerical software. But even for calculating the inductance of a single coil, creating the input data file to define the design is tedious, particularly if the coil has many windings. Moreover, for solving an inverse problem, the calculations must be repeated many times, so that the total computation time may become long. Hence, although they may lead to precise results, numerical methods are impractical for solving reverse problems.

A considerable improvement is offered by the Greenhouse method [5]. It allows precise calculations of coil inductance at direct current (DC). It consists of dividing the coil into its constituent straight conductor segments and calculating their partial self-inductance and all mutual inductances between them separately using analytical formulae and summing up all the contributions. But the method doesn't provide an inductance formula that explicitly depends on the design parameters, like e.g. the number of turns, the coil size, etc. Thus, for large numbers of turns and for solving inverse problems, the calculations get tedious.

Therefore, many researchers have worked on finding approximate inductance formulae that explicitly depend on the design parameters. Using such a formula is by far the easiest and fastest way to calculate coil inductance, particularly for solving inverse problems. This approach is expected to produce less accurate results than the Greenhouse method, but it is faster and, above all, much easier to handle. Some formulae approximating the direct current (DC) inductance of square planar spiral coils with rectangular conductor cross section are known from the literature.

Recently, six of the most cited coil inductance formulae [6] - [9] were scrutinized in a comparative study [1] investigating their maximum relative error over a wide domain of definition of the design parameters. The relative errors of the formulae were calculated for nearly 14000 parameter value combinations as the relative deviations of the inductances from precise reference values computed numerically. The maximum relative error was calculated as the maximum modulus of the relative deviations. The main conclusion was that "Further research in finding better formulae is highly encouraged. A generally applicable formula with good accuracy has not been found yet, despite the claims made to the contrary by some of the authors." ([1], p. 47). This paper aims at deriving such a formula.

Subsequent inspection of the extensive data collected during the course of [1] has revealed that the relative errors of the inductance formula of [9], after correcting an erroneous equation, seem to correlate with the number of windings $N$ and the filling factor $\rho$ (which is a normalized measure of the 
extent to which the area taken by the coil is used up or covered by its windings). This observation has spurred the hope for improving the formula by amending it by a correction factor depending on $N$ and $\rho$. This hope has been justified even more since $N$ is the most and $\rho$ the second-most influential parameter of the four dimensionless ones determining coil inductance. It has seemed possible to determine the correction factor with the help of a two-dimensional fit to the relative errors of the formula.

This idea has resulted in an improved inductance formula being valid for any coil size and inductance range. It reduces the maximum error over the whole domain of definition from $29 \%$, the current state of the art, as was substantiated in [1], down to $2.0 \%$. This has been tested with the same method and reference data used in [1].

In section 2 the original inductance formula of [9] is derived. Section 3 derives the modified formula. Section 4 presents the results of the error analysis, comparing the maximum relative errors of all six formulae from the comparative study with the error of the new one. A comparison with measurements is presented in section 5. Section 6 describes a MATLAB source code for implementing the formula (given in the appendix), together with numerical examples. Section 7 concludes the paper.

\section{Derivation of Jenei's inductance formula}

Because Jenei et al. missed to give a comprehensive derivation of their formula ([9], equation (7)), this section makes up for it. Fig. 1 shows the layout of a square planar spiral coil upon which they based their formula, together with the geometric definition of the design parameters, which are:

- $\quad N$, number of turns or windings, $N \geq 2$.

- $A$, outermost mid-conductor side length.

- $A_{i}$, innermost mid-conductor side length.

- $\quad w$, winding distance or -pitch $(w=s+g)$.

- $g$, gap or spacing between windings.

- $s$, conductor width.

- $h$, conductor height or -thickness, hidden in Fig. 1.

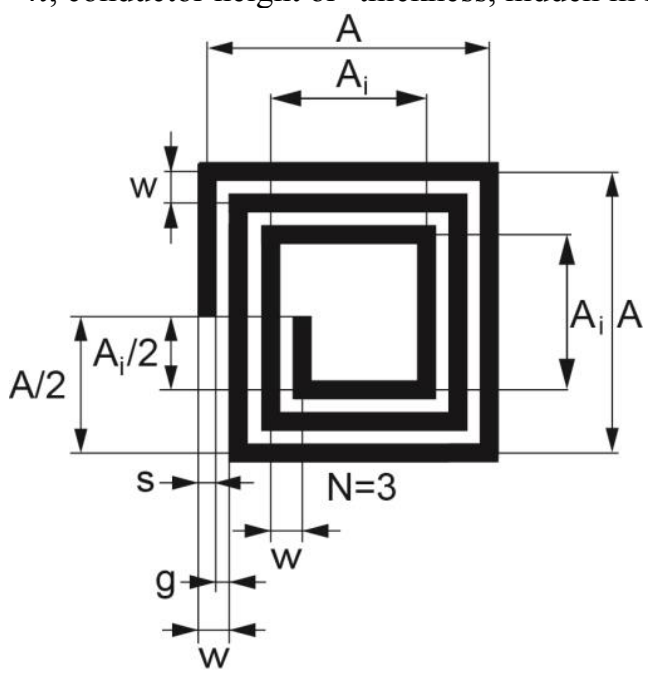

Figure 1: The layout of a square planar spiral coil and its design parameters.
The partial inductance of a coil be defined as the sum of the partial self-inductances of its $4 N$ straight conductor segments (counting the two shorter rim segments of lengths $A / 2$ and $A_{i} / 2$ as one). Let the average length of all segments be $l_{a v}$. With the help of the total length $l$,

$$
l=4 N l_{a v}
$$

the partial coil inductance can then be written in the general form ([10], equation (33))

$$
\begin{aligned}
L_{\text {partial }}=\frac{\mu_{0} l}{2 \pi}[ & \log \left(\sqrt{l_{a v}{ }^{2}+A M S D^{2}}+l_{a v}\right)-\log G M D \\
& \left.-\sqrt{1+\left(\frac{A M S D}{l_{a v}}\right)^{2}}+\frac{A M D}{l_{a v}}\right]
\end{aligned}
$$

Throughout this paper, log designates the natural logarithm. The constant $\mu_{0}$ is the magnetic permeability of the vacuum, $\mu_{0}=4 \pi \cdot 10^{-7} \mathrm{Vs} /(\mathrm{Am})$. The exact expression for the logarithm of the geometric mean distance (GMD) between two arbitrary points in the cross-sectional plane of a conductor with rectangular cross section is known but complicated ([11], § 692 (6)). It has been shown ([12], p. 314 and [13], p. 22 ) how the GMD can be approximated to better than $0.5 \%$,

$$
G M D=0.2235(s+h),
$$

or, equivalently, to the same precision of the GMD,

$$
\log G M D=\log (s+h)-3 / 2 .
$$

Equation (3) is nearly exact for a conductor of infinite crosssectional aspect ratio $s / h$. With its help and with the arithmetic mean square distance (AMSD) and the arithmetic mean distance (AMD) neglected against $l_{a v}$, equation (2) reduces to

$$
L_{\text {partial }}=\frac{\mu_{0} l}{2 \pi}\left[\log \left(\frac{2 l_{a v}}{s+h}\right)+\frac{1}{2}\right]
$$

Expressing $l_{a v}$ in terms of $l$ with the help of equation (1) and isolating $\log (1 / 2)=-0.69315$, equation (4) becomes

$$
L_{\text {partial }}=\frac{\mu_{0} l}{2 \pi}\left\{\log \left[\frac{l}{N(s+h)}\right]-0.19315\right\} \text {. }
$$

To get the total inductance of a coil, the mutual inductances of its parallel conductor segments must also be considered. The mutual inductances of segments at a right angle vanish. In [9] the mutual inductance of two parallel conductor segments separated by the central distance $d$ was approximated by the mutual inductance of their central filaments. It is also given by equation (2), but with $l_{a v}$ instead of $l$ in the first factor, and with a different value of the GMD. Further, the mean distances AMSD and AMD can no longer be neglected. For filaments, they are all identical to $d$ : 


$$
\left\{\begin{array}{c}
G M D=d \\
A M S D=d \\
A M D=d .
\end{array}\right.
$$

The central filaments approximation (6) holds the better the longer $l_{a v}$ is compared to the cross-sectional dimensions (here $s$ and $h$ ), as was shown in [10] for conductors of circular cross section. This is true for all cross sections.

Two types of mutual inductances occur in square planar spiral coils: There are those of a segment on one side of the square and any one on the opposite side, and there are those of a segment on one side and any remaining one on the same side. They can all be approximated by two average values: $\mathrm{m}^{-}$for pairings of segments on opposite sides of the square, and $\mathrm{m}^{+}$for pairings of segments on the same side.

The average mutual inductance $m^{-}$results from equation (2) as described above, with the mean distances according to equations (6), where $d=l_{a v}$, since the average length of the segments is identical to the mean distance between such on opposite sides of the square, see Fig. 1. Thus, we have

$$
m^{-}=\frac{\mu_{0} l_{a v}}{2 \pi}[\log (\sqrt{2}+1)-\sqrt{2}+1]=\frac{\mu_{0} l_{a v}}{2 \pi} 0.46716 .
$$

Each of the $N$ horizontal segments on the upper side of the square couples with each of the $N$ ones on the lower side, and vice versa. This leads to $2 N^{2}$ mutual inductances $m^{-}$. And each of the $N$ vertical segments on the left-hand side of the square couples with each of the $N$ ones on the right-hand side, and vice versa. This yields another $2 N^{2}$, or $4 N^{2}$ in total. Now Jenei et al. use composite mutual inductances of the form $\mathrm{M}^{-}=4 \mathrm{Nm}^{-}$. By equation (1) this becomes

$$
M^{-}=\frac{\mu_{0} l}{2 \pi}[\log (\sqrt{2}+1)-\sqrt{2}+1]=\frac{\mu_{0} l}{2 \pi} 0.46716 .
$$

Hence, there remain $N$ composite mutual inductances $M^{-}$. Since the current in segments on opposite sides of the square flows in opposite directions, they must be subtracted from the partial inductance: $-\mathrm{NM}^{-}$.

The average mutual inductance $m^{+}$is also given by equation (2) with $l$ in the first factor replaced by $l_{a v}$, and likewise the mean distances according to equations (6). Here, $d=d^{+}$, the mean distance between segments on the same side of the square, must be used,

$$
\begin{aligned}
m^{+}=\frac{\mu_{0} l_{a v}}{2 \pi}\left\{\log \left[\sqrt{1+\left(\frac{l_{a v}}{d^{+}}\right)^{2}}+\frac{l_{a v}}{d^{+}}\right]\right. \\
\left.-\sqrt{1+\left(\frac{d^{+}}{l_{a v}}\right)^{2}}+\frac{d^{+}}{l_{a v}}\right\},
\end{aligned}
$$

where $d^{+}$is given by

$$
d^{+}=\frac{(N+1)}{3} w
$$

The mathematical proof of equation (8) can be found in appendix 1. Each of the $N$ horizontal segments on the upper side of the square couples with each of the remaining $N-1$ ones on the same side. This leads to $N(N-1)$ mutual inductances $m^{+}$. (Herein, the mutual inductances in the reverse coupling direction are already included). For the four sides of the square, we get $4 N(N-1)$. Jenei et al. use composite mutual inductances of the form $\mathrm{M}^{+}=4 \mathrm{Nm}^{+}$. By equation (1) this becomes, if the remaining $l_{a v}$ in $m^{+}$are also expressed in terms of $l$,

$$
\begin{aligned}
M^{+}=\frac{\mu_{0} l}{2 \pi} & \left\{\log \left[\sqrt{1+\left(\frac{l}{4 N d^{+}}\right)^{2}}+\frac{l}{4 N d^{+}}\right]\right. \\
& \left.-\sqrt{1+\left(\frac{4 N d^{+}}{l}\right)^{2}}+\frac{4 N d^{+}}{l}\right\} .
\end{aligned}
$$

Therefore, there remain $N-1$ composite mutual inductances $\mathrm{M}^{+}$. Since the current in segments on the same side of the square flows in the same direction, they must be added to the partial inductance: $+(N-1) M^{+}$. So, the total inductance $L_{0}$ is given by

$$
L_{0}=L_{\text {partial }}-N M^{-}+(N-1) M^{+} .
$$

With the help of equations (5), (7), (9), and (10), and by reducing the precision of the two numerical constants, one arrives at Jenei et al.'s original notation

$$
\begin{array}{r}
L_{0}=\frac{\mu_{0} l}{2 \pi}\left\{\log \left(\frac{l}{N(s+h)}\right)-0.2-0.47 N+(N-1)\right. \\
\\
\quad\left\{\operatorname { l o g } \left[\sqrt{\left.1+\left(\frac{l}{4 N d^{+}}\right)^{2}+\frac{l}{4 N d^{+}}\right]}\right.\right. \\
\left.\left.-\sqrt{1+\left(\frac{4 N d^{+}}{l}\right)^{2}}+\frac{4 N d^{+}}{l}\right\}\right\} .
\end{array}
$$

The total length $l$ can be calculated in terms of the design parameters $N, A_{i}$, and $w$. Note that its original expression ([9], equation (1)) turned out to be false [1]. The correct one was proven to be ([1], equations (23), (31), (32), and the mathematical proof in the appendix)

$$
l=4 N A_{i}+\left(4 N^{2}-5 N\right) w,
$$

where

$$
A_{i}=A-2(N-1) w .
$$

Equation (13) can easily be verified with the help of Fig. 1. 


\section{Derivation of the modified inductance formula}

The first step towards improving the inductance formula of [9] had already been taken in the comparative study [1] by deriving the correct equation (12) for the total length of the conductor. With its help, the maximum errors of equation (11) dropped significantly, at least for the two lower ranges: Compare the first two values in Table 1 with those of [9] in Table 2 .

The maximum relative errors seemed to correlate with the ranges, see Table 1. More precisely, they seemed to correlate with $N$, whose value distinguishes the ranges (see below). The two-windings range didn't need any correction. As for the three upper ones, the possible correlation could only be exploited for improving accuracy if it was the result of a large systematic error combined with a smaller amplitude of statistical fluctuations. In other words, the errors had to be biased, either towards positive or negative values.

Table 1: The maximum relative errors of equation (11) with $l$ evaluated with the help of equation (12) for the four ranges defined in this section.

\begin{tabular}{cccc}
\hline $\begin{array}{c}\text { Two-wind. } \\
{[\%]}\end{array}$ & Low range & Med. range & High range \\
{$[\%]$} & {$[\%]$} & {$[\%]$} \\
\hline 1.7 & 9.3 & 18 & 24 \\
\hline
\end{tabular}

A closer inspection of the large data compiled as part of [1] revealed that this was the case. Further, it had been found that $N$ was the most and the filling factor $\rho$ the second-most influential parameter of the four dimensionless ones determining coil inductance ([1], Table 1). Hence, it was obvious to extend the quest for correlation also to $\rho$ to improve precision. Indeed, the errors not only correlated with $N$, but also with $\rho$, leaving some minor statistical fluctuations caused by the variation of the remaining dimensionless parameters $\kappa$ and $\gamma$ (see below). This meant that formula (11) could effectively be improved by adjoining a correction factor depending on $N$ and $\rho$ to compensate for the systematic error. The correction factor might be found with the help of a two-dimensional fit to the relative errors of equation (11). All errors were negative, except for one parameter combination with $N=3$ (for which the error was so small that even a slight overcompensation due to the wrong sign of the correction wouldn't harm.)

Equation (11) was derived from physical principles, so it is scalable [1]. This means that it scales linearly upon stretching or shrinking all dimensional parameters $A, w, s$, and $h$ with a constant factor. Consequently, the formula holds for all coil sizes and for any inductance range, since scalability is a general property of coil inductance. For a proof in the case of planar spiral coils, see ([1], p. 39). The modified formula will remain scalable, even though the correction factor will be based on a data fit. This is because the correction factor will only depend on dimensionless parameters $(N$ and $\rho)$. Note that the filling factor $\rho$ does not appear in equation (11). It can be expressed as ([1], equation (7))

$$
\rho=\frac{(N-1) w+s}{A-(N-1) w} \text {. }
$$

In [1] the coil designs were described by combinations of values of dimensionless parameters. This was to benefit from the reduction of the parameter space dimension from five to four. The remaining two dimensionless parameters, besides $N$ and $\rho$, were the relative winding distance $\kappa$ defined as

$$
\kappa=\frac{w}{s}>1
$$

and the cross-sectional aspect ratio $\gamma$,

$$
\gamma=\frac{s}{h} \geq 1
$$

The condition $\gamma \geq 1$ reflected a constraint on printed circuit boards (PCB's) and on IC's. Herewith, the set of equations transforming dimensional to dimensionless parameters was complete. The inverse transformation equations can be found in ([1], equations (12) - (15)).

In the comparative study, the precise inductances of 13851 coil designs were calculated [1]. The designs were defined by $A=1 \mathrm{~mm}$ and all parameter combinations given by the Cartesian products of the sets of values of the dimensionless parameters $N, \rho, \kappa$, and $\gamma$ listed below. The precise reference inductances were calculated with the help of the free standard software FastHenry2 [14]. It can be downloaded from the site www.fastfieldsolvers.com. The calculations were done at $\mathrm{DC}$, requesting $2 \times 2$ subfilaments. For the conductivity of copper at $20^{\circ} \mathrm{C}$, the value $\sigma=5.9595 \cdot 10^{7} \Omega^{-1} \mathrm{~m}^{-1}$, corresponding to the resistivity $1 / \sigma=1.678 \cdot 10^{-8} \Omega \mathrm{m}$, was used $([15], T=293 \mathrm{~K})$. The sets of parameter sampling values were

Two-windings range: $N=2$,

$\rho=0.01,0.0537,0.0975,0.1412,0.1850,0.2288,0.2725$, $0.3162,0.36$

Low range: $N=3,4,5,6,7$, $\rho=0.01,0.0737,0.1375,0.2013,0.2650,0.3287,0.3925$, $0.4563,0.52$.

Medium range: $N=8,9,10,11,12$, $\rho=0.01,0.1063,0.2025,0.2988,0.3950,0.4913,0.5875$, $0.6838,0.78$

High range: $N=13,14,15,16,17,18,19,20$, $\rho=0.01,0.1162,0.2225,0.3287,0.4350,0.5413,0.6475$, $0.7538,0.86$.

All ranges:

$\kappa=1.1,2.2125,3.3250,4.4375,5.5500,6.6625,7.7750$, $8.8875,10$.

$\gamma=1,2.4,5.6,13.3,31.6,75,177.8,422,1000$.

The same parameter combinations and the resulting precise reference inductances were used in the present work to derive the modified inductance formula (25), as explained below and in the error analysis described in section 4 . 
Note that the values of $\rho$ differ from range to range. The reason is that, depending on the minimum number of $N$ in a range and the maximum value $\kappa=10$, a maximum value of $\rho$ is allowed. This is to prevent invalid parameter combinations, for which the length of one of the constituent straight conductor segments would vanish or even become negative ([1], p. 40).

For the three upper ranges and each of their values of $\rho$, a linear function of $N$ was by far good enough to be fitted to the relative errors $\Delta L / L$ of equation (11) in function of $N$ :

$$
\frac{\Delta L}{L}(N)=c_{1} N+c_{2}
$$

For each value of $\rho$, the values of the constants $c_{1}$ and $c_{2}$ differed. For the two-windings range, they were set to zero because it didn't need any correction, see Table 1 . For the three upper ranges, doing the linear fit (15) for the 9 sampling values of $\rho$ resulted in 9 values of the constants $c_{1}$ and $c_{2}$. By replacing the constants with fitting functions $c_{1}(\rho)$ and $c_{2}(\rho)$, the two-dimensional fit to the relative error $\Delta L / L$ of equation (11) in function of $N$ and $\rho$ was completed to

$$
\frac{\Delta L}{L}(N, \rho)=c_{1}(\rho) N+c_{2}(\rho) .
$$

The functions $c_{1}(\rho)$ and $c_{2}(\rho)$ were determined by fitting them to the 9 values of the constants $c_{1}$ and $c_{2}$, respectively. It was found that for all three ranges and both constants, a $1^{\text {st }}$ order rational function worked perfectly, with one exception: For $c_{2}$ in the low range, a $2^{\text {nd }}$ order polynomial performed better.

The coefficients of a rational function can always be scaled by presetting one of them to unity. Here, it was the zero-order coefficient of the denominator polynomial, see equations (19) and (21) - (24). Thus, only three coefficients were still unknown, both in the rational functions and in the polynomial (20).

These fits represented two independent linear optimization problems of dimension three solvable in the least-squares sense by means of an overdetermined system of linear equations. The fits worked best for $\kappa=1.1$ and $\gamma=1$ left constant. (Of course, in the error analysis, all four parameters $N$, $\rho, \kappa$, and $\gamma$ will be varied, see section 4 ).

It turned out that the relative errors of the modified inductance formula (25), with the functions $c_{1}(\rho)$ and $c_{2}(\rho)$ based on coefficients resulting from the least-squares fits, were still biased: The extreme positive error didn't match the extreme negative one. In the high-range, the extreme positive error was $+0.7 \%$, while the extreme negative one was $-5.4 \%$, instead of $\pm[(0.7 \%-(-5.4 \%)] / 2= \pm 3.05 \%$ if the errors were balanced.

This bias could, in principle, be removed by directly minimizing the maximum error of the inductance resulting from equation (25), instead of the least-squares errors of the fitting functions $c_{1}(\rho)$ and $c_{2}(\rho)$. (In fact, this way the extreme errors could even be lowered further, down to $\pm 2.0 \%$, see section 4). Only, this no longer represented a linear optimization problem. Moreover, the coefficients of the functions $c_{1}(\rho)$ and $c_{2}(\rho)$ could no longer be computed separately, because they did not belong to two independent optimization problems anymore, but to one and the same. So, they had to be determined simultaneously. This led to a global non-linear optimization problem of dimension six (for the three unknown coefficients of each of the two functions $c_{1}(\rho)$ and $\left.c_{2}(\rho)\right)$.

It was found that this global optimization problem could be reduced to a local one by taking the solutions to the linear problem as starting values for the non-linear one. The coefficients solving the linear problem were systematically multiplied by the five factors $0.5,0.75,1.0,1.5$, and 2.0 in six nested loops, with one loop for each of the six coefficients. In the innermost loop, the relative errors of equation (25), with the functions $c_{1}(\rho)$ and $c_{2}(\rho)$ based on the respective coefficients, were calculated for all designs, and both the extreme positive and the extreme negative error were stored together with the six respective coefficients in eight columns of a matrix, with one line for each of the $5^{6}=15625$ combinations of coefficients. This was done for all three upper ranges separately. One of the 15625 combinations of coefficients was then picked as the solution. Namely, the one for which the magnitude of both the extreme positive and the extreme negative error were minimal, in which case they were also balanced. This procedure minimized the maximum error of the inductance calculated by equation (25).

Thus, the search for six coefficients in the infinite interval $(-\infty, \infty)$ was reduced to one of six factors in the finite interval $[0.5,2.0]$, and even further to five discrete factors. It turned out that the rather coarse mesh of coefficients generated by the five discrete factors was good enough. This simple method for solving the global optimization problem, approximately but systematically, proved to be a viable compromise between computing time and accuracy. On a personal computer with $4 \mathrm{GHz}$ clock frequency, the procedure took 4 hours and 22 minutes (where the precise reference inductances were already known from [1]). The resulting functions $c_{1}(\rho)$ and $c_{2}(\rho)$, normalized to fit to the percentaged negative errors of equation (11), were found to be:

Two-windings range:

$$
\begin{aligned}
& c_{1}(\rho) \equiv 0, \\
& c_{2}(\rho) \equiv 0 .
\end{aligned}
$$

Low range:

$$
\begin{gathered}
c_{1}(\rho)=-\frac{7.2 \rho+0.35}{2.78 \rho+1}, \\
c_{2}(\rho)=-12.8 \rho^{2}+11 \rho+0.80 .
\end{gathered}
$$

Medium range:

$$
\begin{aligned}
& c_{1}(\rho)=-\frac{2.1 \rho+0.17}{0.75 \rho+1}, \\
& c_{2}(\rho)=-\frac{0.59 \rho+1.2}{-0.90 \rho+1} .
\end{aligned}
$$


High range:

$$
\begin{aligned}
& c_{1}(\rho)=-\frac{1.5 \rho+0.11}{0.98 \rho+1}, \\
& c_{2}(\rho)=-\frac{2.85 \rho+2.48}{-0.75 \rho+1} .
\end{aligned}
$$

Note that equations (22) and (24) are both singular at some point $\rho>1$. However, these points lie beyond the domain of definition $0<\rho<1$ ([1], equation (6)). Hence, in the domain of definition, the functions (19) - (24) are exempt from singularities. With the help of equation (16), the modified, improved inductance formula reads

$$
L=L_{0}\left(1-\frac{c_{1}(\rho) N+c_{2}(\rho)}{100}\right)
$$

$L_{0}$ is given by equations (5) and (7) - (10) or, equivalently, by equation (11), but with the precise constants 0.19315 and 0.46716 . The filling factor $\rho$ is given by equation (14), and the functions $c_{1}(\rho)$ and $c_{2}(\rho)$ are expressed by equations (17) - (24). Equations (17) and (18) guarantee that, for the two-windings range, $L \equiv L_{0}$.

\section{Error analysis}

In the comparative study, it was concluded that "The method used in the present study for the error analysis may also prove useful for standardized error tests on future formulae. Using the same method, domains of definition, and sampling values as presented in section 3 will guarantee that only geometrically valid designs are considered, that all parameter space dimensions are scanned homogeneously, and that the results will be comparable to those reported in section 4 of this paper." ([1], p. 47). Consequently, in the present work, the error analysis of equation (25) was done in the same way as described in [1], by varying all four dimensionless parameters $N, \rho, \kappa$, and $\gamma$. In other words, the relative errors of equation (25) were calculated for all four ranges or 13851 parameter combinations defined in section 3 .

Table 2 compares the maximum errors of equation (25) with those of the formulae from the literature for the four ranges ([1], Tables 5-9 and 11). The results show that equation (25) improves the precision of inductance calculations by means of a formula substantially, compared to what was possible with the best formula so far. This was $29 \%$ in the two-windings range, $23 \%$ in the low range, $13 \%$ in the medium range, and $8.8 \%$ in the high range, or a maximum error of $29 \%$ over all ranges. With equation (25), the maximum error over all ranges collapses to $2.0 \%$.

Note that due to $L \equiv L_{0}$ in the two-windings range, the maximum error of equation (11) in Table 1 should be identical to the one of equation (25) in Table 2 . Yet, the former is $1.7 \%$, while the latter is $1.9 \%$. The reason for the difference is that for the former, the rounded values of the constants, i.e. 0.2 and 0.47, were used, as in [9] and [1]. By chance, this gave a slightly smaller maximum error. For all other ranges, the precise constants led to a smaller maximum error.
Table 2: The maximum relative errors of the formulae from the literature together with those of equation (25) for the four ranges defined in section 3 .

\begin{tabular}{ccccc}
\hline Formula & $\begin{array}{c}\text { Two-wind. } \\
{[\%]}\end{array}$ & $\begin{array}{c}\text { Low } \\
{[\%]}\end{array}$ & $\begin{array}{c}\text { Med. } \\
{[\%]}\end{array}$ & $\begin{array}{c}\text { High } \\
{[\%]}\end{array}$ \\
\hline$[6]$ & 51 & 63 & 67 & 69 \\
{$[7]$} & 33 & 23 & 23 & 30 \\
{$[8], 1$} & 39 & 37 & 35 & 34 \\
{$[8], 2$} & 29 & 23 & 13 & 8.8 \\
{$[8], 3$} & 35 & 26 & 22 & 22 \\
{$[9]$} & 101 & 70 & 15 & 14 \\
Eq. $(25)$ & 1.9 & 1.8 & 2.0 & 2.0 \\
\hline
\end{tabular}

\section{Comparison with measurements}

Measurements on 16 RFID reader antennas were performed with an Agilent ${ }^{\mathbb{R}}$ 4294A Precision Impedance Analyzer with a 42941A Impedance Probe. It had already been shown in [16] that two-wire transmission lines, i.e. circuits of closely spaced conductors, exhibit a spurious increase of the reactance at high frequencies resulting from LC resonance. This is no different in planar spiral coils, where the conductor segments also lie close together and locally form spurious capacitors. This phenomenon impedes the precise measurement of the inductance at $13.56 \mathrm{MHz}$. For this reason, it was measured at $300 \mathrm{kHz}$ instead, representing the DC value. The marginal reduction of the inductance at $13.56 \mathrm{MHz}$ compared to DC as shown in Table 4 warranted the validity of this procedure.

Table 3 lists the 16 reader antennas manufactured on PCB with standard copper layer thickness $h=35 \mu \mathrm{m}$, numbered in the first column (\#) and characterized by their design parameters $N, A, w$ (in mils), and $s$ (both in mils and in millimeters. 1 mil corresponds to $25.4 \mu \mathrm{m}$ ).

Table 3: Dimensional design parameters of 16 reader anten-

\begin{tabular}{cccccc}
\multicolumn{5}{c}{ nas manufactured on standard PCB $(h=35 \mu \mathrm{m})}$. \\
\hline$\#$ & $N$ & $\begin{array}{c}A \\
{[\mathrm{~mm}]}\end{array}$ & $\begin{array}{c}w \\
{[\mathrm{mil}]}\end{array}$ & $\begin{array}{c}S \\
{[\mathrm{mil}]}\end{array}$ & $\begin{array}{c}S \\
{[\mathrm{~mm}]}\end{array}$ \\
\hline 1 & 3 & 20 & 12 & 6 & 0.15 \\
2 & 3 & 20 & 20 & 6 & 0.15 \\
3 & 3 & 20 & 30 & 6 & 0.15 \\
4 & 3 & 20 & 40 & 6 & 0.15 \\
5 & 3 & 20 & 30 & 12 & 0.30 \\
6 & 3 & 50 & 12 & 6 & 0.15 \\
7 & 3 & 50 & 20 & 6 & 0.15 \\
8 & 3 & 50 & 40 & 6 & 0.15 \\
9 & 3 & 50 & 20 & 12 & 0.30 \\
10 & 3 & 50 & 30 & 12 & 0.30 \\
11 & 3 & 50 & 40 & 12 & 0.30 \\
12 & 4 & 50 & 40 & 12 & 0.30 \\
13 & 5 & 50 & 40 & 12 & 0.30 \\
14 & 3 & 100 & 40 & 12 & 0.30 \\
15 & 5 & 10 & 12 & 6 & 0.15 \\
16 & 3 & 50 & 30 & 18 & 0.46 \\
\hline
\end{tabular}


Table 4: Precise DC inductance $L_{\text {precise }}$ of the antennas from Table 3, the relative errors of the measurements, of equation (25), and the relative reduction of the inductance at $13.56 \mathrm{MHz}$ compared to DC.

\begin{tabular}{ccccc}
\hline$\#$ & $\begin{array}{c}L_{\text {precise }} \\
{[\mathrm{nH}]}\end{array}$ & $\begin{array}{c}\text { Err. } \\
\text { meas. } \\
{[\%]}\end{array}$ & $\begin{array}{c}\text { Err. } \\
(25) \\
{[\%]}\end{array}$ & $\begin{array}{c}\text { Red. } \\
{[\%]}\end{array}$ \\
\hline 1 & 542 & 0.15 & -0.52 & -0.89 \\
2 & 480 & 1.14 & -0.17 & -0.75 \\
3 & 426 & 2.13 & 0.12 & -0.74 \\
4 & 385 & 1.29 & 0.37 & -0.77 \\
5 & 401 & 1.09 & -0.09 & -1.27 \\
6 & 1716 & 0.19 & -0.55 & -0.73 \\
7 & 1575 & 0.51 & -0.38 & -0.60 \\
8 & 1372 & 0.90 & -0.15 & -0.59 \\
9 & 1510 & 0.16 & -0.69 & -1.16 \\
10 & 1391 & 0.40 & -0.41 & -0.99 \\
11 & 1305 & 0.33 & -0.26 & -0.95 \\
12 & 2037 & 0.26 & -0.45 & -0.81 \\
13 & 2849 & 0.40 & -0.47 & -0.72 \\
14 & 3173 & 0.13 & -0.38 & -0.81 \\
15 & 446 & 0.50 & -0.47 & -0.87 \\
16 & 1352 & 0.01 & -0.67 & -1.34 \\
\hline
\end{tabular}

Table 4 presents the same coil designs as Table 3 , in the same order, with their precise DC inductance $L_{\text {precise }}$ calculated with the help of the software FastHenry2. It also includes the relative errors of the measurements and of equation (25). The measurement errors agreed well with the manufacturer's specifications of the measurement set-up. According to Fig. $10-6$ of the operation manual, the error at $300 \mathrm{kHz}$ was less than $1 \%$ for inductances above about $1 \mu \mathrm{H}$, and less than $3 \%$ below it.

The error of equation (25) never exceeded $0.7 \%$, in accordance with the maximum of $2.0 \%$ reported in section 4 .

At high frequencies, both the skin and the proximity effect lower the inductance of the constituent straight conductor segments of the coil, and thus its total inductance, compared to DC [16], [17]. To investigate the strength of the combined effect at $13.56 \mathrm{MHz}$, numerical calculations were done with the help of the software FastHenry2, now requesting $10 \times 10$ subfilaments to allow for nonhomogeneous current distribution due to the skin- and the proximity effect. The last column of Table 4 shows the relative reduction of the inductance at $13.56 \mathrm{MHz}$ compared to DC. The largest reduction occurred at \#16 and amounted to $-1.34 \%$. Increasing the number of subfilaments to $15 \times 15$ changed it only marginally to $-1.33 \%$. So, it was concluded that $10 \times 10$ subfilaments sufficed to compute the reduction of the inductance due to frequency effects at $13.56 \mathrm{MHz}$.

\section{Implementation in MATLAB}

The source code of the MATLAB function L_SquarePlanarspiral to calculate the DC inductance of square planar spiral coils with the help of equation (25) is given in appendix 2. Since the code is short and simple, engineers who don't have access to MATLAB can easily translate it into any other computer language. All quantities are in SI units. Besides the inductance $L$, the function also returns the filling factor $\rho$. If the data entered represents an invalid parameter combination as explained in section 3, an error message is output. This condition is detected by checking the value of $\rho$, which is calculated with the help of equation (14). Following are examples to test the correct implementation of the code for each range. The dimensional coil data

$A=0.05 ; \quad w=1.4 e-3 ; s=7 e-4 ; h=35 e-6 ;$

is used for all four ranges. The MATLAB function is run by the command

$[\mathrm{L}$, rho $]=\mathrm{L}_{\text {S SquarePlanarSpiral }}(\mathrm{N}, \mathrm{A}, \mathrm{W}, \mathrm{s}, \mathrm{h})$

Table 5 lists the number of windings $N$ to be used for each of the four ranges in the test, together with the respective value of $L$ returned by the MATLAB function, the precise DC inductance, the relative error of $L$, and the value of $\rho$ returned.

Table 5: Example data for testing the MATLAB function presented in appendix 2.

\begin{tabular}{ccccc}
\hline$N$ & $\begin{array}{c}L \\
{[\mu \mathrm{H}]}\end{array}$ & $\begin{array}{c}L_{\text {precise }} \\
{[\mu \mathrm{H}]}\end{array}$ & $\begin{array}{c}\text { Err. } \\
{[\%]}\end{array}$ & $\rho$ \\
\hline 2 & 0.595 & 0.595 & -0.05 & 0.0432 \\
5 & 2.354 & 2.366 & -0.51 & 0.1419 \\
10 & 5.402 & 5.406 & -0.07 & 0.3556 \\
15 & 7.011 & 7.013 & -0.02 & 0.6678 \\
\hline
\end{tabular}

\section{Conclusions}

An improved formula for the DC inductance of square planar spiral coils with rectangular conductor cross section has been derived. It is based on purely physical principles on one part, and on a correction factor on the other. The latter has been determined by a parameter fit. In doing so, a simple but effective method for reducing a global non-linear optimization problem to a local one, by using the solution to a related linear problem as starting point for the non-linear one, has been successfully used. Since the correction factor only depends on dimensionless parameters, the derived formula remains scalable, i.e. it is valid irrespectively of the coil size and the inductance range.

The formula has been tested by the same method applied in a previous comparative study [1] which analyzed the maximum errors of the most cited formulae known from the literature over a large domain of definition considering almost 14000 reference designs. Their precise inductances were computed numerically with the help of the scientific and industrial standard software FastHenry2. Using the same method and the same reference designs in this paper guarantees that the test results from the new formula can directly be compared to those reported in that study.

The formula reduces the maximum error of the DC inductance over the whole domain of definition from hitherto $29 \%$ down to $2.0 \%$. Hence, it seems that the long sought-after generally applicable formula with sufficient accuracy for coil design has been found.

Calculations of the DC inductance done on 16 RFID reader antennas manufactured on standard PCB with $35 \mu \mathrm{m}$ copper 
layer thickness have led to a maximum error of the formula of $0.7 \%$, which is compatible with the above result of its maximum error over the whole domain of definition. Measurements performed on these antennas have confirmed the calculations.

Yet, there remain two restrictions in applying the formula, particularly to RFID transponder card antennas:

Firstly, these are usually not square, but rectangular due to the card standard ISO 7810. Hence, it would be useful to extend the formula to include rectangular coils as well. This will add a fifth dimensionless parameter to the set, making the error analysis much costlier.

Secondly, transponder card antennas are often manufactured from round wire. Therefore, it would be desirable to repeat the analysis suggested above for rectangular coils once again, but this time with circular conductor cross section.

Finally, a MATLAB source code for the formula together with numerical examples have been provided.

\section{Appendix 1}

Proof of equation (8):

For $N=3$, there are 2 pairings of adjacent segments separated by the distance $w$, and 1 pairing of segments separated by a distance $2 w$, see Fig. 1 .

In general, for $N=n$, there are $n-1$ pairings of adjacent segments separated by the distance $w, n-2$ pairings of segments separated by a distance $2 w, n-3$ pairings of segments separated by a distance $3 w$, etc., and $n-(n-1)=1$ pairing of segments separated by a distance $(n-1) w$. So, there are

$$
N_{d}=1+2+3+\cdots+n-1=\sum_{k=1}^{n-1} k=\frac{n(n-1)}{2}
$$

distances. The average distance is the sum over all distances divided by the number of distances. The sum over all distances is given by the sum of the above list,

$$
S_{d}=\sum_{k=1}^{n-1}(n-k) k w=n w \sum_{k=1}^{n-1} k-w \sum_{k=1}^{n-1} k^{2} .
$$

The first sum on the r.h.s. is again the sum of the natural numbers, resulting in $n^{2}(n-1) w / 2$. The second sum on the r.h.s. is the sum of squares of the natural numbers, resulting in $n(n-1)(2 n-1) w / 6$, see [18]. Subtracting this from the first sum yields $S_{d}=n\left(n^{2}-1\right) w / 6$, and we find $d^{+}=$ $S_{d} / N_{d}=(n+1) w / 3$, q.e.d.

\section{Appendix 2}

Source code of the MATLAB function L_SquarePlanarSpiral:

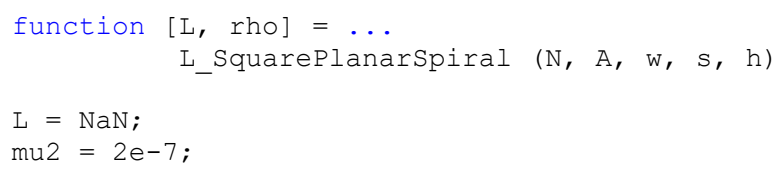

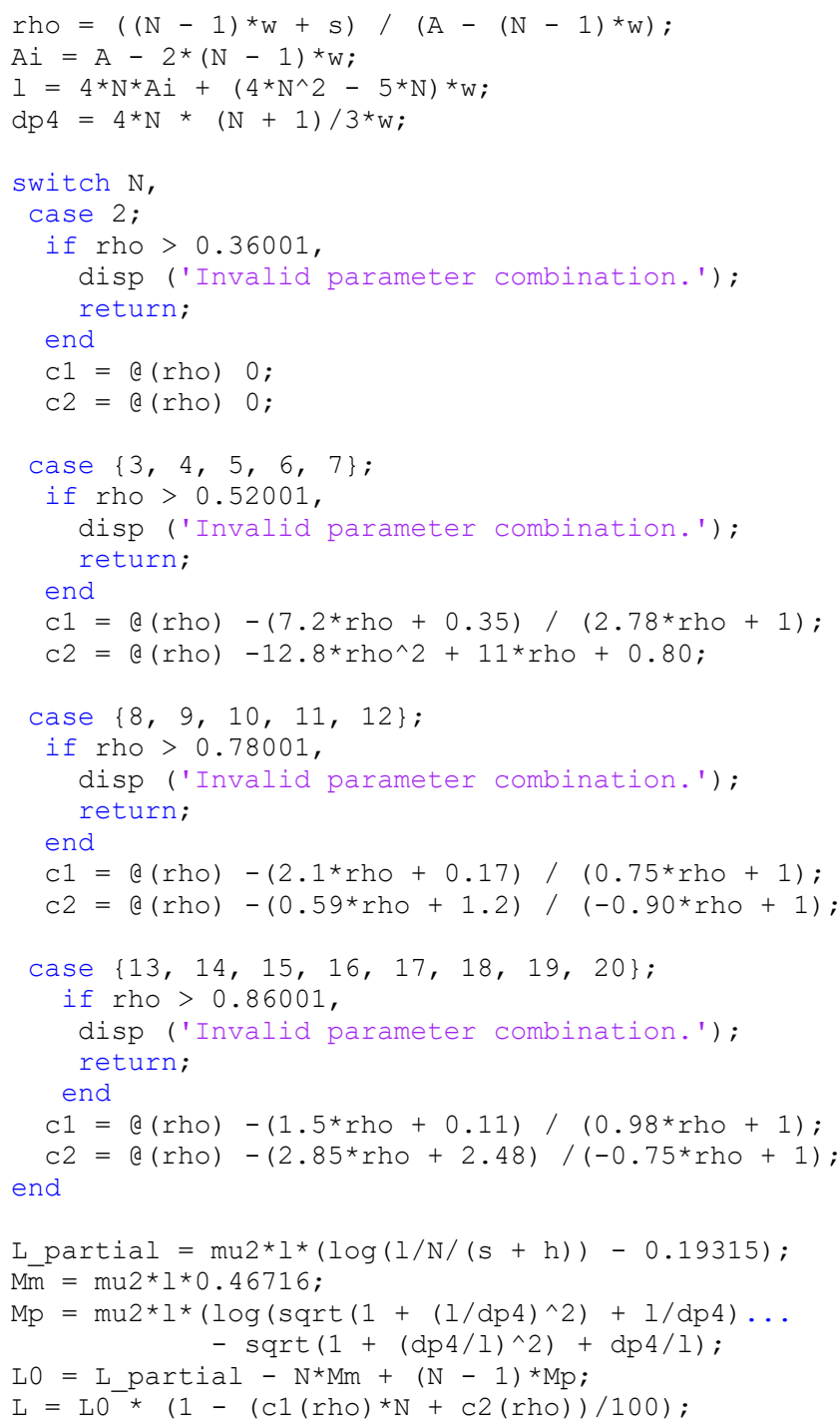

\section{Acknowledgements}

The author wishes to thank LEGIC Identsystems AG for granting the time to work on this project.

\section{References}

[1] H. A. Aebischer, Comparative Study of Analytical Inductance Formulae for Square Planar Spiral Inductors, Advanced Electromagnetics, vol. 7, no. 5, 37-48, 2018. DOI: https://dx.doi.org/10.7716/aem.v7i5.862.

[2] J. Chen and J. J. Liou, On-Chip Spiral Inductors for RF Applications: An Overview, Journal of Semiconductor Technology and Science, vol. 4, no. 3, 149-167, 2004.

[3] D. Paret, RFID and Contactless Smart Card Applications, John Wiley \& Sons, Ltd, West Sussex, 2005.

[4] R. A. Potyrailo, C. Surman, S. Go, Y. Lee, T. Sivavec, and W. G. Morris, Development of radio-frequency identification sensors based on organic electronic sensing materials for selective detection of toxic vapors, Journal of Applied Physics, 106, 124902-1 - 124902-6, 2009. 
[5] H. M. Greenhouse, Design of Planar Rectangular Microelectronic Inductors, IEEE Trans. on Parts, Hybrids, and Packaging, vol. 10, no. 2, 101-109, 1974.

[6] J. Crols, P. Kinget, J. Craninckx, and M. Steyaert, An Analytical Model of Planar Inductors on Lowly Doped Silicon Substrates for High Frequency Analog Design up to $3 \mathrm{GHz}$, IEEE Symposium on VLSI Circuits, Honolulu, Digest of Technical Papers, 28-29, 1996.

[7] H. Ronkainen, H. Kattelus, E. Tarvainen, T. Riihisaari, M. Andersson, and P. Kuivalainen, IC compatible planar inductors on silicon, IEE Proc. Circuits Devices Syst., vol. 144, no. 1, 29-35, 1997.

[8] S. S. Mohan, M. del Mar Hershenson, S. P. Boyd, and T. H. Lee, Simple Accurate Expressions for Planar Spiral Inductances, IEEE Journal of Solid-State Circuits, vol. 34, no. 10, 1419-1424, 1999.

[9] S. Jenei, B. K. J. C. Nauwelaers, and S. Decoutere, Physics-Based Closed-Form Inductance Expression for Compact Modeling of Integrated Spiral Inductors, IEEE Journal of Solid-State Circuits, vol. 37, no. 1, 77-80, 2002.

[10] H. A. Aebischer and B. Aebischer, Improved Formulae for the Inductance of Straight Wires, Advanced Electromagnetics, vol. 3, no. 1, 31-43, 2014. DOI: http://dx.doi.org/10.7716/aem.v3i1.254.

[11] J. C. Maxwell, A Treatise on Electricity and Magnetism, vol. 2., Dover Publications, New York, 1954, unabridged $3^{\text {rd }}$ ed. of 1891.

[12] E. B. Rosa, The self and mutual inductances of linear conductors, Bulletin of the Bureau of Standards, vol. 4, no. 2, Washington, 1907.

[13] F. W. Grover, Inductance Calculations: Working Formulas and Tables, Dover Publications, New York, 2004, first published by D. Van Nostrand Co., New York, 1946.

[14] M. Kamon, M. J. Tsuk, and J. K. White, FASTHENRY: A Multipole-Accelerated 3-D Inductance Extraction Program, IEEE Trans. on Microwave Theory and Techniques, vol. 42, no. 9, 1750-1758, 1994.

[15] W. M. Haynes, Th. J. Bruno, and D. R. Lide, CRC Handbook of Chemistry and Physics, $95^{\text {th }}$ ed., Internet Version 2015, p. 12-41, 2015.

[16] H. A. Aebischer and H. Friedli, Analytical Approximation for the Inductance of Circularly Cylindrical TwoWire Transmission Lines with Proximity Effect, $A d$ vanced Electromagnetics, vol. 7, no. 1, 25-34, 2018 , DOI: http://dx.doi.org/10.7716/aem.v7i1.516.

[17] H. A. Aebischer and B. Aebischer, The GMD Method for Inductance Calculation Applied to Conductors with Skin Effect, Advanced Electromagnetics, vol. 6, no. 2, 77-92, 2017, DOI: http://dx.doi.org/10.7716/aem.v6i2.464.
[18] https://en.wikipedia.org/wiki/Square_pyramidal_number. 